\title{
Zurstiege, Guido: Taktiken der Entnetzung. Die Sehnsucht nach Stille im digitalen Zeitalter
}

\author{
Berlin: Suhrkamp 2019. 297 Seiten. Preis: $€ 18,00$
}

\section{Christian Pentzold}

Online publiziert: 6 . Oktober 2020

(C) Der/die Autor(en) 2020

Keine Bewegung ohne Gegenbewegung. Es scheint eine mediengeschichtliche Konstante, dass technologischer Fortschritt und kommunikative Innovationen stets traditionalistische oder fortschrittspessimistische Reaktionen auslösen - angefangen bei der Ablehnung der Schrift über die Skepsis gegenüber fotografischen Reproduktionen bis hin zur Verweigerung von Smartphones. In dieser Logik einer dialektisch verlaufenden Medien- und Gesellschaftsentwicklung folgen auf Vernetzungsprozesse gegenläufige Initiativen der Entnetzung. Diese sind Gegenstand des vorliegenden Buches. Gegen den Imperativ der zunehmenden Kommunikation, intensiveren Konnektivität und gesteigerten Mediatisierung beobachtet Guido Zurstiege Formen des medienbezogenen Verzichts, der Medienabstinenz bzw. der Selbstverteidigung gegen überhandnehmende Erreichbarkeitszwänge.

Es fällt nicht leicht, das Buch in geläufige wissenschaftliche Formate einzuordnen. Die Abhandlung hat einiges gemeinsam mit Publikationen von public intellectuals aus dem angelsächsischen Raum: sie schaltet sich in ein Thema von gesellschaftlicher Relevanz und Aktualität ein, es berücksichtigt tagesaktuelle Debatten und verarbeitet wissenschaftliche Studien, ohne aber selbst auf einem extensiven empirischen Forschungsvorhaben nach den Regeln des Fachs zu gründen oder sich in das Korsett eines buchgewordenen Projektberichts zu fügen. Im Grunde ist der Band Pathologie und Therapie zugleich; gegen Lärm setzt er Stille, gegen Kontrollverlust zeigt er Wege des Kontrollgewinns auf.

Seine Einsichten zum Unbehagen an der aktuellen Medienkultur hat der Autor erklärtermaßen an jahrelanger Selbstbeobachtung, bei aufmerksamer Lektüre und in persönlichen Gesprächen gewonnen. Sie werden verknüpft mit vornehmlich sozialund kommunikationswissenschaftlicher Forschung zum Medienverzicht und zum

Prof. Dr. C. Pentzold ( $₫)$

Universität Leipzig, Leipzig, Deutschland

E-Mail: christian.pentzold@uni-leipzig.de 
bewusst-reflektierten Mediengebrauch bis hinein ins 19. Jahrhundert. Theoretische Orientierung liefert de Certeaus Unterscheidung in die Strategien gesellschaftlicher Eliten und dominanter Institutionen einerseits und die eigensinnigen bis subversiven Taktiken „,von unten“ andererseits.

Zurstiege behandelt verschiedene Facetten gegenwärtiger Mediennichtnutzung und den damit verknüpften, sehr unterschiedlich gelagerten Gründen. Medienverzicht kann situativ oder langfristig sein; bewusst oder unbewusst geschehen; generell alle digitalen Angebote oder nur einzelne vernetzte Technologien bzw. Kommunikationsformen betreffen. Dabei macht Zurstiege deutlich, dass ein Komplettausstieg Fiktion ist, und er zeigt auf, dass die Misere vielfältig ist. Das Bedürfnis nach Rückzug aus einer medial überreichen, von kommunikativen Angeboten und Diensten durchdrungenen Lebenswelt stellt sich immer drängender angesichts einer Litanei von Herausforderungen: Zwang zur Selbstdarstellung und der permanenten Präsenz auf Plattformen, allgegenwärtige Überwachung, Hassrede und digitale Propaganda, Verschwörungstheorien, Internetsucht, etc.

In seiner Diagnose gelingen Zurstiege eine Vielzahl inspirierender Einsichten, etwa zum performativen Selbstwiderspruch der User, die zwar im Grunde Facebook, Google und Amazon ablehnen, aber zugleich ihre willigen Kund:innen sind. Weitere Ambivalenzen fallen auf, etwa wenn zum einen davon die Rede ist, dass Nutzende bemüht sind, ein möglichst kontrolliertes Bild von sich abzugeben, sie zum anderen aber nicht der Versuchung widerstehen können, auf Twitter Beleidigungen und mancherlei Absurditäten in die Welt zu setzen.

Fraglich ist, ob alle im Buch gemachten Beobachtungen nachvollziehbar sind. Trifft es zum Beispiel zu, dass die Leute ihrer Computertechnik und den Oberflächen des Smartphones Aufmerksamkeit schenken, oder sind es nicht doch die darüber verfügbaren Dienste, die sie mit anderen Bekannten bzw. Unbekannten verknüpfen sowie mit mehr Inhalten versorgen? Auch ob WhatsApp oder Snapchat in der Tat die über sie getätigten Aktionen vergessen, mag bezweifelt werden. Überdies wäre zu überlegen, ob Stille und Schweigen passende Kategorien sind, um die im Buch behandelten Formen des Verzichts und der Subversion bündig zu fassen. Stille herrscht mithin auch, wenn Stimmen unterdrückt werden oder Sprachlosigkeit nicht zu überwinden ist. Zurstiege selbst weist auf den metaphorischen „Lärm der Zeit“" (S. 215) hin und es wäre zu prüfen, inwiefern unter Stille und Schweigen auch die von ihm erkundeten Phänomene medial induzierter Erschöpfung, Überwältigung oder des Verdrusses fallen.

Zentral für das Buch ist das Argument, dass kommunikative (Selbst-)Begrenzung nicht Passivität oder Apathie bedeuten, sondern Medienverzicht unter Anstrengung gewonnen wird sowie beständig aufrechterhalten und verteidigt werden muss. Die durch Verzicht gewonnene Freiheit, Aufmerksamkeit und Zeit bleiben nicht ungenutzt, sondern sollen für ein Mehr an Kreativität, Produktivität oder sozialen Beziehungen eingesetzt werden. Digitale Medien üben keinen Zwang aus, unbedingt oder notwendigerweise benutzt zu werden; vielmehr emulieren sie alltägliche Tätigkeiten und verflechten sich mit ihnen. In Anlehnung an Böll spricht Zurstiege von einer „fürsorglichen Belagerung“ (S. 26).

Nur am Rande geht Zurstiege darauf ein, dass mediale Enthaltsamkeit ebenso ein Privileg ist wie kommunikative Teilhabe. Wenn er von „Positionierung durch 
Verzicht“"(S. 18) spricht, zeigt er auf, dass sich Distinktionsgewinne nicht nur durch die Wahl bestimmter Medienprodukte erzielen lassen, wie von Bourdieu vorgedacht, sondern auch durch bewusste und als solche dargestellte enthaltsame Selbstbestimmung. Zugleich aber könnte der Rückgriff auf Bourdieu die vielfältigen Dispositionen deutlich machen, welche die Chance auf Medienverzicht bedingen. Wie die umfangreiche Forschung zur Verfügbarkeit von Zeit zeigt, sind Möglichkeiten zum medialen Widerstand und zur Entkopplung aus medienbedingten Arbeitsverhältnissen und Sozialbeziehungen sehr ungleich vorhanden und werden durch Herkunft, Geschlecht, Vermögen und Position definiert. Die in dem Buch gelieferte luzide Erfassung von kommunikativem Stress und medialem Overload sollte deshalb nicht den Blick darauf verstellen, dass die empfohlene Gelassenheit und Langsamkeit nicht allein Geisteshaltungen sind, sondern zum Teil mühevoll erwirkt werden bzw. unerreichbar bleiben. Erschöpfung mag allgemeines Gefühl sein, Erholung aber kostet. Gerade weil diese wichtigen Fragen im Raum stehen, ist dem anregenden Band ein breites Publikum zu wünschen.

Funding Open Access funding enabled and organized by Projekt DEAL.

Open Access Dieser Artikel wird unter der Creative Commons Namensnennung 4.0 International Lizenz veröffentlicht, welche die Nutzung, Vervielfältigung, Bearbeitung, Verbreitung und Wiedergabe in jeglichem Medium und Format erlaubt, sofern Sie den/die ursprünglichen Autor(en) und die Quelle ordnungsgemäß nennen, einen Link zur Creative Commons Lizenz beifügen und angeben, ob Änderungen vorgenommen wurden.

Die in diesem Artikel enthaltenen Bilder und sonstiges Drittmaterial unterliegen ebenfalls der genannten Creative Commons Lizenz, sofern sich aus der Abbildungslegende nichts anderes ergibt. Sofern das betreffende Material nicht unter der genannten Creative Commons Lizenz steht und die betreffende Handlung nicht nach gesetzlichen Vorschriften erlaubt ist, ist für die oben aufgeführten Weiterverwendungen des Materials die Einwilligung des jeweiligen Rechteinhabers einzuholen.

Weitere Details zur Lizenz entnehmen Sie bitte der Lizenzinformation auf http://creativecommons.org/ licenses/by/4.0/deed.de.

Prof. Dr. Christian Pentzold ist Professor für Medien- und Kommunikationswissenschaft an der Universität Leipzig. 